2008

\title{
Kilovolt Blumlein Pulse Generator With Variable Pulse Duration and Polarity
}

Andrea de Angelis

Old Dominion University

Juergen F. Kolb

Old Dominion University

Luigi Zeni

Karl H. Schoenbach

Old Dominion University

Follow this and additional works at: https://digitalcommons.odu.edu/bioelectrics_pubs

Part of the Electrical and Electronics Commons, and the Systems and Communications Commons

\section{Repository Citation}

de Angelis, Andrea; Kolb, Juergen F.; Zeni, Luigi; and Schoenbach, Karl H., "Kilovolt Blumlein Pulse Generator With Variable Pulse Duration and Polarity" (2008). Bioelectrics Publications. 230.

https://digitalcommons.odu.edu/bioelectrics_pubs/230

\section{Original Publication Citation}

de Angelis, A., Kolb, J. F., Zeni, L., \& Schoenbach, K. H. (2008). Kilovolt Blumlein pulse generator with variable pulse duration and polarity. Review of Scientific Instruments, 79(4), 044301. doi:10.1063/1.2901609

This Article is brought to you for free and open access by the Frank Reidy Research Center for Bioelectrics at ODU Digital Commons. It has been accepted for inclusion in Bioelectrics Publications by an authorized administrator of ODU Digital Commons. For more information, please contact digitalcommons@odu.edu. 


\title{
Kilovolt Blumlein pulse generator with variable pulse duration and polarity
}

\author{
Andrea de Angelis, ${ }^{1,2,3}$ Juergen F. Kolb, ${ }^{3}$ Luigi Zeni, ${ }^{1}$ and Karl H. Schoenbach ${ }^{3}$ \\ ${ }^{1}$ Department of Information Engineering, Second University of Naples, Via Roma 29, I-81031 Aversa, Italy \\ ${ }^{2}$ Dipartimento di Informatica, Matematica, Elettronica e Trasporti (DIMET), Università Mediterranea di \\ Reggio Calabria, Località Feo di Vito, I-89060 Reggio Calabria, Italy \\ ${ }^{3}$ Frank Reidy Research Center for Bioelectrics, Old Dominion University, 830 Southampton Ave., Norfolk, \\ Virginia 23510, USA
}

(Received 21 January 2008; accepted 3 March 2008; published online 9 April 2008)

\begin{abstract}
A Blumlein pulse generator which utilizes the superposition of electrical pulses launched from two individually switched pulse forming lines has been designed and tested. By using a power metal-oxide-semiconductor field-effect transistor as a switch on each end of the Blumlein line, we were able to generate pulses with amplitudes of $1 \mathrm{kV}$ across a $100 \Omega$ load. Pulse duration and polarity can be controlled by the temporal delay in the triggering of the two switches. Using this technique, we have demonstrated the generation of pulses with durations between 8 and $60 \mathrm{~ns}$. The lower limit in pulse duration was determined by the switch closing time and the upper limit by the length of the pulse forming line. A further advantage of the concept is that pulse distortions caused by the non-negligible on-resistance of a line with a single switch can be eliminated by using switches with identical characteristics. (C) 2008 American Institute of Physics.
\end{abstract}

[DOI: $10.1063 / 1.2901609$ ]

\section{INTRODUCTION}

A pulse generator concept that is widely used for the generation of high voltage pulses is based on the propagation of electric signals in pulse forming lines (PFLs) or their pulse forming network (PFN) analogs, which are built from discrete components. One particular type, named after its inventor, Blumlein, ${ }^{1}$ provides flat (rectangular) voltage pulses with an amplitude equal to the charging voltage $V_{0}$ delivered into a load with a resistance $R_{L}$, with a value matching twice the impedance of the individual pulse forming lines $Z_{0}$ (Fig. 1 , configuration with switch $S_{1}$ only). The concept is described in detail elsewhere. ${ }^{2,3}$ The Blumlein pulse generator utilizes a single closing switch, and the pulse duration is determined by the electrical and geometrical properties of the PFL. Any variation of the pulse duration requires changing the length of the transmission lines or, for PFNs, changing its components. Besides this practical limitation, the pulses generated are also affected by the switch performance. For switches such as metal-oxide-semiconductor field-effect transistors (MOSFETs), where the nominal on-state resistance of several ohms results in a forward voltage upon reflection of an incident electrical signal, the measured voltage across the load does not decrease to zero after the main pulse, ${ }^{3}$ but remains (for a time comparable to the main pulse duration) at a non-negligible level. This can be up to $20 \%$ of the pulse amplitude, despite the load being perfectly matched to the impedance of the pulse generator. ${ }^{4}$

This effect can be understood by considering the phenomena at the switch end of the configuration (Fig. 1, with switch $S_{1}$ only). The voltage across the load arises from a potential difference between the signals propagating along the branches on either side of the load. For impedance matching $R_{L}=2 Z_{0}$ between load and pulse generator, a pulse amplitude equal to the charging voltage of the system results from a voltage step of $-V_{0} / 2$ propagating toward the switch and a second step of $+V_{0} / 2$ propagating toward the open end.

The voltage stays at this level for the time it takes the signals to be reflected at the switch and at the open end, respectively, and for both to return to the load where they superimpose, to zero, finally discharging the system across the load. This portrayal assumes that, ideally, the signals launched from the switch are reflected at a short circuit at this end, i.e., that the resistance of the switch is zero when closed.

If, however, the switch resistance in the single switch configuration is not zero, the step reflected from the switch will have lower amplitude than the step approaching from the open end. This asymmetry leads to an incomplete termination of the pulse when the signals return to the load (after twice the transit time of a single PFN). As result, pulse amplitudes are lower than expected and the remaining potential differences propagating along the system lead to a residual voltage difference across the load after the pulse.

For real switches, this problem cannot be solved in a classic single switch system, but only minimized by employing low resistance switches. It poses a limitation in the use of fast MOSFETs, not only in a single switch configuration but also in series configuration ${ }^{5}$ since the overall resistance increases.

The practical shortcomings of a fixed pulse duration and of nonideal real closing switches can be overcome by a concept recently introduced, which utilizes two independently controllable switches to terminate the Blumlein assembly on either end. It permits one to vary pulse duration and pulse polarity without physical changes to the arrangement. ${ }^{6}$ In 


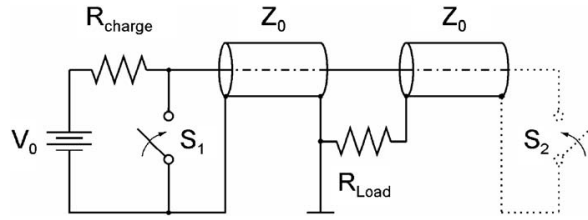

FIG. 1. Circuit diagram of the basic Blumlein pulse-forming system with one closing switch only and the modified system having a second identical closing switch at the other end of the pulse forming line (dotted line).

addition, the circuit, which is shown in Fig. 1 (with switches $S_{1}$ and $S_{2}$ ), allows us to avoid any pulse distortions caused by the finite switch resistance in the on-state. In this modified Blumlein configuration, instead of relying on reflections from ideally closed and open ends, respectively, pulses propagating from both ends toward the load are generated by identical, but not necessarily ideal, closing switches. As a result, symmetric differences in the waves propagating from the switch toward the load are assimilated in the superposition of waves at the load.

In the following, we will demonstrate the suppression of reflection for this case through modeling and show experimental results for a circuit assembled using two identical commercially available semiconductor switches (MOSFETs).

\section{CIRCUIT SIMULATION RESULTS}

Simulation results for this new type of Blumlein pulse generator (Fig. 1 with both switches $S_{1}$ and $S_{2}$ ), presented first in reference 6 , were based on the assumption that the switches are ideal, meaning that they have an infinitely large resistance before switching, which is reduced to zero instantaneously upon closing of the switch. However, a more realistic approach to modeling requires taking into account the finite resistance of closing switches in the on state.

To understand the effect of the switch resistance, a SPICE simulation has been performed taking into account a constant and finite resistance of $5 \Omega$ for the switches in the on state. The closing time of the switches was assumed to be $1 \mathrm{~ns}$. The two $50 \Omega$ transmission lines with a length corresponding to a transit time of $30 \mathrm{~ns}$ are matched with a $100 \Omega$ load. Both transmission lines are considered lossless. This model was applied to both the Blumlein circuit with a single switch (Fig. 1, switch $S_{1}$ only) as well as to the modified circuit with two switches (Fig. 1, switches $S_{1}$ and $S_{2}$ )

The results are shown in Fig. 2(a) for the Blumlein circuit with a single switch and in Fig. 2(b) for the dual switch system. In the first case, the length of the transmission lines was chosen to generate a $60 \mathrm{~ns}$ long pulse. For the dual switch configuration, the second switch was set to close $40 \mathrm{~ns}$ after the first switch resulting in a $40 \mathrm{~ns}$ pulse duration for the same transmission lines.

In both cases, when the switch closes, the voltage across the switch $S_{1}$ decreases to $V_{S}=0.5 V_{0}\left[1+\left(R_{S}-Z_{0}\right) /\left(R_{S}+Z_{0}\right)\right]$, with $R_{S}$ as the residual on resistance of the switch. (For an ideal switch with $R_{S}=0$, the voltage would be zero.) For a charging voltage of $V_{0}=1 \mathrm{kV}$, together with an assumed switch on resistance of $R_{S}=5 \Omega$ and for a transmission line

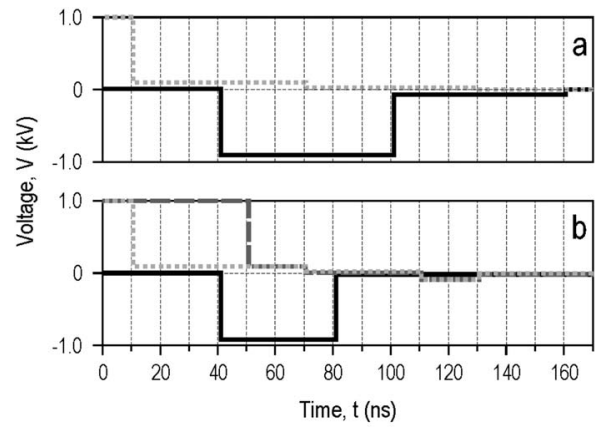

FIG. 2. SPICE simulation results for Blumlein-type circuits with switches with a resistance of $5 \Omega$ in the on state. (a) Basic Blumlein pulse-forming system (single switch): the temporal development of the voltage across the switch (doted line) and the load (solid line) is shown; the pulse duration is $60 \mathrm{~ns}$, determined by twice the transit time of an electromagnetic wave along a single PFL (transmission line cable). (b) Modified Blumlein pulseforming system (dual switch system). In the upper section, the temporal development of the voltage across the first (doted line) and second (dashed line) switch is shown. The temporal development of the voltage across the load is shown in the lower section (solid line). The pulse duration of $40 \mathrm{~ns}$ is determined by the delay in triggering switch $S_{2}$ with respect to switch $S_{1}$. Note that the voltage after the pulse has returned to zero, different from the case with the single switch (a).

impedance $Z_{0}=50 \Omega$, this residual voltage across the closed switch is $91 \mathrm{~V}$ or approximately $9 \%$ of the charging voltage.

Conversely, the voltage step launched from the switch into the transmission line is reduced to $91 \%$ of its ideal value, and consequently, will superimpose after $30 \mathrm{~ns}$ (transit time of the pulse along the left transmission line) on the ideal signal propagating from the open ended transmission line toward the load for a voltage difference of $V_{L}=\left(0.5 V_{0}-V_{S}\right)$ $+0.5 V_{0}=0.5 V_{0}\left[1-\left(R_{S}-Z_{0}\right) /\left(R_{S}+Z_{0}\right)\right], \quad$ in our example, $90.9 \%$ of the charging voltage $V_{0}$ or $909 \mathrm{~V}$.

After $60 \mathrm{~ns}$, which corresponds to the roundtrip time for the propagation of the voltage step launched from the end of an individual line to the load and back, the voltage at switch $S_{1}$ is again reduced to $91 \%$ or to $8.2 \mathrm{~V}$ in the example here. This is assuming that the switch on resistance stays constant. With every subsequent passage between switch and load the residual voltage across switch and load is reduced further in the same way, eventually approaching zero.

For the symmetric setup of the configuration modified with a second closing switch of the same resistive characteristic, voltage steps of the same amplitude are launched toward the load. In our example, the second switch is triggered to close $40 \mathrm{~ns}$ after the first switch [Fig. 2(b)]. In the meantime, the signal arriving from the first switch after $30 \mathrm{~ns}$ again leads to a voltage difference of $909 \mathrm{~V}$ across the load, which is maintained until the signal sent toward the load from the second switch is superimposed, bringing the potential difference across the load effectively to zero after 40 ns.

The use of real, identical switches on either end of the Blumlein configuration solves the problem of a lingering voltage being applied to the load after the end of the intended pulse duration. In addition, the duration of the pulse can be varied by setting different delays between the trigger signals for both switches. Changing the order in which the switches are triggered changes the polarity of the pulse across the 

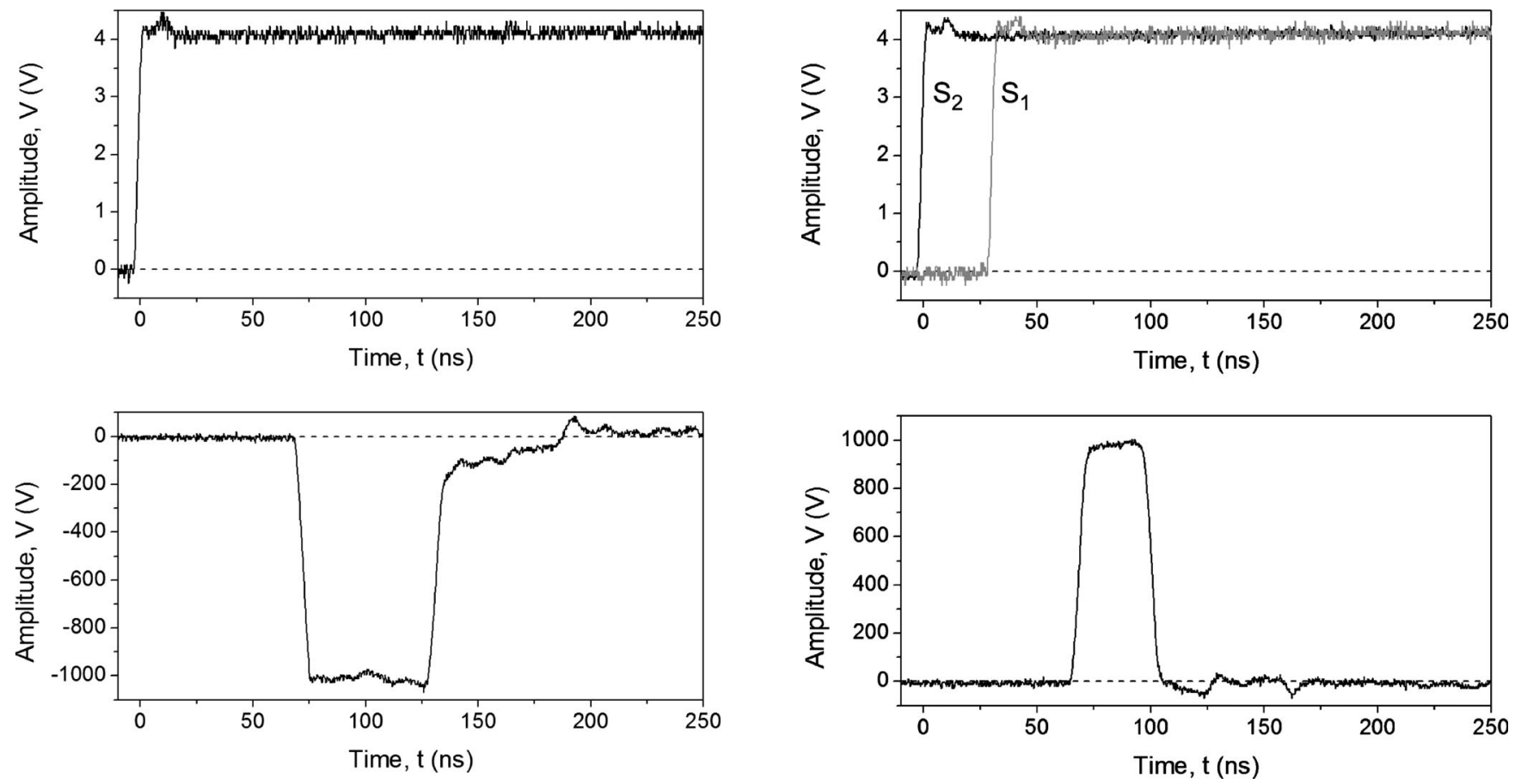

FIG. 3. Experimental result: standard Blumlein configuration. The trace in the upper section shows the temporal development of the trigger signal, that in the lower section the voltage across the $100 \Omega$ load resistor. Note the residual voltage after the main pulse.

load. However, because of the finite on resistance of the switches, the pulse amplitude will be lowered by the residual voltage across a single switch.

\section{EXPERIMENTAL RESULTS}

In order to demonstrate the effect of the dual switch Blumlein, we have used $50 \Omega$ coaxial cables (RG58) of $6 \mathrm{~m}$ length as PFLs on either side of a $100 \Omega$ load. This pulse generator, if used with a single switch (Fig. 1), is expected to generate a $60 \mathrm{~ns}$ long pulse with an amplitude equal to the applied voltage (assuming an ideal switch and a lossless transmission line). As switches we have used power MOSFETs (IXYS DE275-102N06A) which can hold a voltage of $1 \mathrm{kV}$, have a closing time of about $2 \mathrm{~ns}$, and a nominal residual resistance in the on state of $R=1.6 \Omega$.

In Fig. 3, the trigger signal and the voltage across the load for the classical Blumlein configuration, recorded by means of a voltage probe (Tektronix Passive HV Probes model P5100), are shown as they appear on a digital oscilloscope (Tektronix, TDS3052B). The voltage across the load reaches almost the full applied voltage of $1 \mathrm{kV}$, which is in agreement with a simulated reduction of $31 \mathrm{~V}$. After $60 \mathrm{~ns}$

FIG. 4. Experimental result: modified Blumlein configuration. The traces in the upper section show the temporal development of the trigger signals for switches 1 (solid) and 2 (dashed), respectively. The trace in the lower section shows the temporal development of the voltage across the $100 \Omega$ load resistor (determined by the delay time between switch triggering). The voltage pulse does not show the kind of distortion seen with single switch Blumlein devices. Note that by triggering switch $S_{2}$ before switch $S_{1}$, the polarity has been inverted.

(full width at half maximum), it decreases to approximately $100 \mathrm{~V}$, which is almost three times as much as expected for

a residual switch resistance of $1.6 \Omega$, gradually decreasing to about $50 \mathrm{~V}$ during the following $60 \mathrm{~ns}$. The measurements suggest that the on resistance of the MOSFET changes with time approaching the value stated in the specifications only after $120 \mathrm{~ns}$. In order to demonstrate the suppression of the residual voltage signal, we measured the voltage across the load in the modified circuit, with a second identical MOSFET switch at the previously open end. To demonstrate that the sequence of triggering determines the polarity, we used an example in which switch $S_{2}$ was triggered before switch $S_{1}$. Figure 4 shows the two trigger signals for switch $S_{1}$ and switch $S_{2}$, respectively, and the pulse measured at the load. The results show that the polarity has changed compared to the traditional Blumlein setup. The pulse duration is determined by $\Delta T=\left|T_{2}-T_{1}\right|$, with $T_{1}$ and $T_{2}$ representing closing times of the switches $S_{1}$ and $S_{2}$, respectively. By introducing the sign function, the voltage difference $V_{L}$ across the load $R_{L}$ can be described according to

$$
V_{L}= \begin{cases}\operatorname{sign}\left(T_{1}-T_{2}\right)\left(V_{0}-V_{S}\right) & \min \left(T_{1}, T_{2}\right)+\tau<t<\min \left(T_{1}, T_{2}\right)+\tau+|\Delta T| \\ 0 & \text { otherwise },\end{cases}
$$


where $V_{0}$ is the charging voltage and $\tau$ is the propagation time of the pulse on the single line.

\section{SUMMARY}

A Blumlein-type pulse generator with two independently triggered switches, instead of a single closing switch, has been developed. Numerical and experimental studies have confirmed initial design considerations. ${ }^{6}$ With this modified Blumlein pulse generator, it is possible to generate rectangular voltage pulses with variable duration, limited for short pulses by the risetime of the switches and for long pulses by the PFN design. As long as the switches are identical, even nonlinear characteristics affecting the pulse shape in the classic Blumlein configuration can be compensated for by the symmetry of the modified system. Moreover, the pulse duration can be controlled externally by a delay in the triggering of switch $S_{2}$ with respect to switch $S_{1}$. Reversing the trigger sequence, switching $S_{2}$ before $S_{1}$ allows us to change the polarity of the pulse for the same charging voltage $V_{0}$. Besides providing a spectrum of pulse durations with a single system, the modified Blumlein-type pulse generator allows us to suppress any residual voltage signals which have their origin in the nonideal behavior (residual resistance) of the closing switches.

One application for such a device is in the field of bioelectrics, where nanosecond pulses with voltages of up to $1 \mathrm{kV}$ are required to perform studies on living biological cells under the microscope. ${ }^{4}$

\section{ACKNOWLEDGMENTS}

This research work was performed with funds provided by the Frank Reidy Research Center for Bioelectrics. Helpful discussions with Professor Giovanni Leone are sincerely acknowledged.

${ }^{1}$ A. D. Blumlein, U.S. Patent No. 2,496,979 (7 February 1950).

${ }^{2}$ P. W. Smith, Transient Electronics (Wiley, Chichester, UK, 2002), Chap. 3.

${ }^{3}$ J. F. Kolb, S. Kono, and K. H. Schoenbach, Bioelectromagnetics 27, 172 (2006).

${ }^{4}$ W. Frey, J. A. White, R. O. Price, P. F. Blackmore, R. P. Joshi, R. Nuccitelli, S. J. Beebe, K. H. Schoenbach, and J. F. Kolb, Biophys. J. 90, 3608 (2006)

${ }^{5}$ R. J. Baker and S. T. Ward, IEE Electronics Lett. 30, 1634 (1994).

${ }^{6}$ A. de Angelis, L. Zeni, and G. Leone, IEE Electronics Lett. 42, 205 (2006). 